(n)

\title{
Miura and auto-Backlund transformations for the $q$-deformed KP and q-deformed modified KP hierarchies \\ Jipeng Cheng
}

To cite this article: Jipeng Cheng (2017) Miura and auto-Backlund transformations for the $q$-deformed KP and $q$-deformed modified KP hierarchies, Journal of Nonlinear Mathematical Physics 24:1, 7-19, DOI:

https://doi.org/10.1080/14029251.2016.1274111

To link to this article: https://doi.org/10.1080/14029251.2016.1274111

Published online: 04 January 2021 


\title{
Miura and auto-Backlund transformations for the $q$-deformed KP and $q$-deformed modified KP hierarchies
}

\author{
Jipeng Cheng \\ Department of Mathematics, China University of Mining and Technology \\ Xuzhou, Jiangsu 221116, P. R. China \\ Email: chengjp@cumt.edu.cn and chengjp@mail.ustc.edu.cn
}

Received 7 September 2016

Accepted 6 October 2016

\begin{abstract}
The Miura and anti-Miura transformations between the $q$-deformed KP and the $q$-deformed modified KP hierarchies are investigated in this paper. Then the auto-Backlund transformations for the $q$-deformed KP and $q$-deformed modified KP hierarchies are constructed through the combinations of the Miura and anti-Miura transformations. And the corresponding results are also generalized to the constrained cases. At last, some examples of Miura and auto-Backlund transformations are given.

Keywords: the $q$-KP and $q$-mKP hierarchies; the constrained $q$-KP and constrained $q$-mKP hierarchies; Miura transformation; auto-Backlund transformation.
\end{abstract}

2010 Mathematics Subject Classification: 35Q51, 37K10, 37K40

\section{Introduction}

The $q$-deformed integrable systems are the important research objects $[1,3-7,11-14,18-24]$ in the theoretical physics and mathematics, which emerge out of the attempt to quantize the classical integrable systems. The $q$-deformed integrable systems are defined by means of $q$-derivative $\partial_{q}$ (see (2.1) in Section 2) instead of usual derivative $\partial$ with respect to $x$ in the classical systems. When $q \rightarrow$ 1 , the $q$-deformed integrable systems will become into the classical systems. There are several kinds of $q$-deformed integrable systems, for example: the $q$-deformed Kadomtsev-Petviashvili ( $q$-KP) hierarchy [1,3,5-7, 19-22], the $q$-deformed modified Kadomtsev-Petviashvili ( $q$-mKP) hierarchy [4,13,18,23], the $q$-deformed AKNS-D hierarchy [24], the $q$-KP hierarchy and the $q$-mKP hierarchy with self-consistent sources [12,13], the $q$-Toda hierarchy [11] and so on. Among these $q$-deformed integrable systems, there is little literature on the $q$-mKP hierarchy. In this paper, we mainly deal with the relations between the $q$-KP and $q$-mKP hierarchies.

The Miura transformation $[15,17]$ plays a vital role in the classical integrable systems, since it reveals the internal connections between different integrable properties such as the solution structures $[9,15]$ and the Hamiltonian structures $[10,16,17]$. There exist the Miura transformations between the KP and mKP hierarchies [17]. The $q$-deformed integrable systems own many similar properties to the classical ones, for example, the tau functions [7], the gauge transformations $[4,6,21]$, the additional symmetries $[19,22]$ and so on. Thus it is natural to ask whether the Miura links exist between the $q$-KP and $q$-mKP hierarchies. In this paper, it is found that there are two kinds of the Miura transformations $T_{\mu}$ and $T_{v}$ (see Table 2 in Section 3), which are the transformations from the solutions of the $q$-mKP hierarchy to the solutions of the $q$-KP hierarchy, and two kinds of anti-Miura transformations $T_{m}$ and $T_{n}$ (see Table 1 in Section 3), which are 
the inverse of the Miura transformations. Then through $q$-KP $\stackrel{\text { anti-Miura }}{\longrightarrow} q$-mKP $\stackrel{\text { Miura }}{\longrightarrow} q$-KP and $q$-mKP $\stackrel{\text { Miura }}{\longrightarrow} q-\mathrm{KP} \stackrel{\text { anti-Miura }}{\longrightarrow} q$-mKP, the auto-Backlund transformations are constructed. Here the auto-Backlund transformations mean the transformations from the $q$-KP hierarchy to the $q$-KP hierarchy, or from the $q$-mKP hierarchy to the $q$ - $\mathrm{mKP}$ hierarchy. And it is discovered that the autoBacklund transformations obtained in this way are consistent with the corresponding results in $[4,6]$. The Miura and auto-Backlund transformations are also investigated in the constrained cases of the $q$-KP and $q$-mKP ( $q$-cKP and $q$-cmKP) hierarchies.

This paper is organized in the following way. In Section 2, the brief reviews about the $q$-KP and $q$-mKP hierarchies are given. Then the Miura and auto-Backlund transformations are constructed for the $q$-KP and $q$-mKP hierarchies in Section 3. In Section 4, the Miura and auto-Backlund transformations are investigated for the constrained $q$-KP and $q$-mKP hierarchies. After that, some examples of the Miura and auto-Backlund transformations are listed in Section 5. At last, some conclusions and discussions are given in Section 6.

\section{2. $q$-KP and $q$-mKP hierarchies}

Firstly, let's introduce some useful formulas [6,7]. The $q$-derivative $\partial_{q}$ and the $q$-shift operator are defined by their actions on a function $f(x)$

$$
\partial_{q}(f(x))=\frac{f(q x)-f(x)}{x(q-1)}, \quad \theta(f(x))=f(q x) .
$$

In this paper, for any $q$-pseduo-differential operator $A=\sum_{i \ll \infty} p_{i} \partial_{q}^{i}$ and any function $f(x), A(f)$ denotes the action of the operator $A$ on the function $f$, while $A f$ or $A \cdot f$ means the multiplication of $A$ and $f$, which obeys the following $q$-deformed Leibnitz rule

$$
\partial_{q}^{n} f=\sum_{k \geq 0}\left(\begin{array}{l}
n \\
k
\end{array}\right) \theta_{q} \theta^{n-k}\left(\partial_{q}^{k}(f)\right) \partial_{q}^{n-k}, \quad n \in \mathbb{Z}
$$

where the $q$-number and the $q$-binomial are defined by

$$
(n)_{q}=\frac{q^{n}-1}{q-1}, \quad\left(\begin{array}{l}
n \\
k
\end{array}\right)_{q}=\frac{(n)_{q}(n-1)_{q} \cdots(n-k+1)_{q}}{(1)_{q}(2)_{q} \cdots(k)_{q}}, \quad\left(\begin{array}{l}
n \\
0
\end{array}\right)_{q}=1 .
$$

Here $\partial_{q}^{-1}$ denotes the formal inverse of $\partial_{q}$. It is noted that $\theta$ does not commute with $\partial_{q}$,

$$
\partial_{q}\left(\theta^{k}(f)\right)=q^{k} \theta^{k}\left(\partial_{q}(f)\right), \quad k \in \mathbb{Z} .
$$

For $A=\sum_{i} p_{i} \partial_{q}^{i}$, we denote $A_{\geq k}=\sum_{i \geq k} p_{i} \partial_{q}^{i}, \quad A_{<k}=\sum_{i<k} p_{i} \partial_{q}^{i}, A_{[k]}=p_{k}$. Denote "**" as the conjugation operator, which is defined through $\partial_{q}^{*}=-\partial_{q} \cdot \theta^{-1}, f^{*}=f$ and $(A B)^{*}=B^{*} A^{*}$.

Then the $q$-KP hierarchy $[6,7]$ is defined in the following Lax equation,

$$
\frac{\partial L_{q K P}}{\partial t_{k}}=\left[\left(L_{q K P}^{k}\right)_{\geq 0}, L_{q K P}\right]
$$

where the Lax operator $L_{q K P}$ is given by

$$
L_{q K P}=\partial_{q}+u+u_{1} \partial_{q}^{-1}+\cdots
$$


$u=u(x, t)=u\left(x, t_{1}, t_{2}, \cdots\right)$ and $u_{i}=u_{i}(x, t)=u_{i}\left(x, t_{1}, t_{2}, \cdots\right)$. For $n=1$ and $n=2$, one can find the infinite number of equations given by

$$
\begin{aligned}
\frac{\partial u}{\partial t_{1}}= & \theta\left(u_{1}\right)-u_{1}, \\
\frac{\partial u_{1}}{\partial t_{1}}= & \partial_{q}\left(u_{1}\right)+\theta\left(u_{2}\right)+u u_{1}-u_{2}-u_{1} \theta^{-1}(u), \\
& \vdots \\
\frac{\partial u}{\partial t_{2}}= & \theta \partial_{q}\left(u_{1}\right)+\theta^{2}\left(u_{2}\right)+\theta(u) \theta\left(u_{1}\right)+u \theta\left(u_{1}\right) \\
& -\left(\partial_{q}\left(u_{1}\right)+u_{1} u+u_{1} \theta^{-1}(u)+u_{2}\right)
\end{aligned}
$$

By eliminating $u_{1}$ and $u_{2}$ in (2.7), (2.8) and (2.9), one can arrive at the $q$-KP equation

$$
(\theta-1)\left(\frac{\partial u}{\partial t_{2}}-2 u \frac{\partial u}{\partial t_{1}}-\frac{\partial^{2} u}{\partial t_{1}^{2}}+\frac{2}{(q-1) x} \frac{\partial u}{\partial t_{1}}\right)=2 \frac{\partial^{2} u}{\partial t_{1}^{2}}
$$

As far as I know, the $q$-KP equation (2.10) is derived for the first time.

The eigenfunction $\Phi$ and the adjoint eigenfunction $\bar{\Phi}$ of the $q$-KP hierarchy are defined as follows

$$
\frac{\partial \Phi}{\partial t_{k}}=\left(L_{q K P}^{k}\right)_{\geq 0}(\Phi), \quad \frac{\partial \bar{\Phi}}{\partial t_{k}}=-\left(L_{q K P}^{k}\right)_{\geq 0}^{*}(\bar{\Phi})
$$

The $q$-KP hierarchy can also be expressed in terms of dressing operator [6,7], that is,

$$
L_{q K P}=W \partial_{q} W^{-1}
$$

where the dressing operator $W$ is given by

$$
W=1+w_{1} \partial_{q}^{-1}+w_{2} \partial_{q}^{-2}+\cdots
$$

and satisfies

$$
\frac{\partial W}{\partial t_{k}}=-\left(L_{q K P}^{k}\right)_{<0} W=-\left(W \partial_{q}^{k} W^{-1}\right)_{<0} W .
$$

Another important integrable hierarchy is the $q$-mKP hierarchy given by the Lax equation $[4,13]$ below

$$
\frac{\partial L_{q m K P}}{\partial t_{k}}=\left[\left(L_{q m K P}^{k}\right)_{\geq 1}, L_{q m K P}\right],
$$

with the Lax operator $L_{q K P}$ defined by

$$
L_{q m K P}=v \partial_{q}+v_{0}+v_{1} \partial_{q}^{-1}+\cdots,
$$


$v=v(x, t)=v\left(x, t_{1}, t_{2}, \cdots\right)$ and $v_{i}=v_{i}(x, t)=v_{i}\left(x, t_{1}, t_{2}, \cdots\right)$. Using the Lax equation (2.15) for $n=1$ and $n=2$, one can find the infinite set of equations

$$
\begin{aligned}
\frac{\partial v}{\partial t_{1}}= & v\left(\theta\left(v_{0}\right)-v_{0}\right), \\
\frac{\partial v_{0}}{\partial t_{1}}= & v \partial_{q}\left(v_{0}\right)+v \theta\left(v_{1}\right)-v_{1} \theta^{-1}(v), \\
& \vdots \\
\frac{\partial v}{\partial t_{2}}= & v \theta(v)\left(\theta \partial_{q}\left(v_{0}\right)-\partial_{q}\left(v_{0}\right)\right)+v \partial_{q}(v)\left(\theta\left(v_{0}\right)-v_{0}\right) \\
& +v \theta\left(v_{0}^{2}\right)-v v_{0}^{2}+v \theta(v) \theta^{2}\left(v_{1}\right)-v \theta^{-1}(v) v_{1},
\end{aligned}
$$

By solving the above set of equations, one can obtain the $q$-mKP equation

$$
\frac{\partial v}{\partial t_{2}}=\frac{\partial^{2} v}{\partial t_{1}^{2}}+\partial_{q}(v) \frac{\partial v}{\partial t_{1}}+\left(2 v \frac{\partial}{\partial t_{1}}-v \theta(v) \partial_{q}-v^{2} \partial_{q}+2 \frac{\partial v}{\partial t_{1}}\right)\left((\theta-1)^{-1} \frac{1}{v} \frac{\partial v}{\partial t_{1}}\right),
$$

where $(\theta-1)^{-1}$ means $-\sum_{i=0}^{\infty} \theta^{i}[8]$.

The eigenfunction $\Psi$ and the adjoint eigenfunction $\bar{\Psi}$ satisfy the identities below,

$$
\frac{\partial \Psi}{\partial t_{k}}=\left(L_{q m K P}^{k}\right)_{\geq 1}(\Psi), \quad \frac{\partial \bar{\Psi}}{\partial t_{k}}=\left(L_{q m K P}^{k}\right)_{\geq 1}^{*}(\bar{\Psi}) .
$$

Similar to the $q$-KP case [7], one can easily prove that there is also the dressing operator in the case of the $q$-mKP hierarchy defined by

$$
L_{q m K P}=Z \partial_{q} Z^{-1}
$$

where $Z$ is given by

$$
Z=z_{0}+z_{1} \partial_{q}^{-1}+z_{2} \partial_{q}^{-2}+\cdots
$$

Here $z_{0}^{-1}$ exists. Then the Lax equation (2.15) is equivalent to

$$
\frac{\partial Z}{\partial t_{k}}=-\left(L_{q m K P}^{k}\right)_{\leq 0} Z=-\left(Z \partial_{q}^{k} Z^{-1}\right)_{\leq 0} Z
$$

Then, let us introduce the $q$-cKP hierarchy and the $q$-cmKP hierarchy. The $q$-cKP hierarchy [6] is defined through restricting the Lax operator of the $q$-KP hierarchy to the following form:

$$
L_{q c K P}=\partial_{q}^{N}+u_{N-1} \partial_{q}^{N-1}+\cdots+u_{1} \partial_{q}+u_{0}+\Phi \partial_{q}^{-1} \bar{\Phi}
$$

which satisfies

$$
\begin{gathered}
\frac{\partial L_{q c K P}}{\partial t_{k}}=\left[\left(L_{q c K P}^{k / N}\right)_{\geq 0}, L_{q c K P}\right], \\
\frac{\partial \Phi}{\partial t_{k}}=\left(L_{q c K P}^{k / N}\right)_{\geq 0}(\Phi), \quad \frac{\partial \bar{\Phi}}{\partial t_{k}}=-\left(L_{q c K P}^{k / N}\right)_{\geq 0}^{*}(\bar{\Phi}) .
\end{gathered}
$$


The Lax operator of the $q$-cmKP hierarchy is defined by

$$
L_{q c m K P}=v_{N} \partial_{q}^{N}+v_{N-1} \partial_{q}^{N-1}+\cdots+v_{1} \partial_{q}+v_{0}+\partial_{q}^{-1} \bar{\Psi},
$$

whose evolution equation is describe by

$$
\begin{aligned}
& \frac{\partial L_{q c m K P}}{\partial t_{k}}=\left[\left(L_{q c m K P}^{k / N}\right)_{\geq 1}, L_{q c m K P}\right], \\
& \frac{\partial \bar{\Psi}}{\partial t_{k}}=-\left(L_{q c m K P}^{k / N}\right)_{\geq 1}^{*}(\bar{\Psi}) .
\end{aligned}
$$

One has to show the system of the $q$-cmKP hierarchy is reasonable, just like the $q$-cKP case [6]. In fact, one can easily prove that the $q$-cmKP hierarchy is self-consistent, which means the negative parts of (2.28) can lead to (2.29) with the help of (2.27) .

In this paper, the Miura transformation is the transformation from the solution $Z$ of the $q$-mKP hierarchy to the solution $W$ of the $q$-KP hierarchy. And the inverse transformation is called the antiMiura transformation. On the other hand, the transformation converting $Z$ to $Z^{\prime}$, or $W$ to $W^{\prime}$ is called the auto-Backlund transformation.

\section{Miura and auto-Backlund transformations for the $q$-KP and $q$-mKP hierarchies}

In this section, we will mainly discuss the Miura and auto-Backlund transformation for the $q$-KP and $q$-mKP hierarchies. Before doing this, the following basic identities on $q$-PDO are needed.

Lemma 3.1. For any q-pseduo-differential operator $A$ and arbitrary functions $\Phi$ and $\bar{\Phi}$, one has the following operator identities:

(1) $\left(\Phi^{-1} A \Phi\right)_{\geq 1}=\Phi^{-1} A_{\geq 0} \Phi-\Phi^{-1} A_{\geq 0}(\Phi)$,

(2) $\left(\partial_{q}^{-1} \bar{\Phi} \cdot A \cdot \bar{\Phi}^{-1} \partial_{q}\right)_{\geq 1}=\partial_{q}^{-1} \bar{\Phi} \cdot A_{\geq 0} \cdot \bar{\Phi}^{-1} \partial_{q}-\partial_{q}^{-1} \cdot A_{\geq 0}^{*}(\bar{\Phi}) \cdot \bar{\Phi}^{-1} \partial_{q}$.

Proof. The proof of the first identity can be found in [4]. As for the second one, according to $\left(\partial_{q}^{-1} B_{\geq 0}\right)_{<0}=\partial_{q}^{-1} \cdot\left(B_{\geq 0}^{*}\right)_{[0]}$ for any $q$-pseduo-differential operator $B$ [21],

$$
\begin{aligned}
& \left(\partial_{q}^{-1} \bar{\Phi} \cdot A \cdot \bar{\Phi}^{-1} \partial_{q}\right)_{\geq 1}=\left(\partial_{q}^{-1} \bar{\Phi} \cdot A_{\geq 0} \cdot \bar{\Phi}^{-1}\right)_{\geq 0} \cdot \partial_{q} \\
= & \partial_{q}^{-1} \bar{\Phi} \cdot A_{\geq 0} \cdot \bar{\Phi}^{-1} \partial_{q}-\left(\partial_{q}^{-1} \bar{\Phi} \cdot A_{\geq 0} \cdot \bar{\Phi}^{-1}\right)_{<0} \cdot \partial_{q} \\
= & \partial_{q}^{-1} \bar{\Phi} \cdot A_{\geq 0} \cdot \bar{\Phi}^{-1} \partial_{q}-\partial_{q}^{-1} \cdot A_{\geq 0}^{*}(\bar{\Phi}) \cdot \bar{\Phi}^{-1} \partial_{q} .
\end{aligned}
$$

Proposition 3.1. There are two kinds of elementary anti-Miura transformations $q-K P \rightarrow q-m K P$, that is, let $L$ be the Lax operator of the $q-K P$ hierarchy, and $\Phi$ and $\bar{\Phi}$ be the eigenfunction and the adjoint eigenfunction of the $q-K P$ hierarchy respectively, and

(1) $L \stackrel{T_{m}(\Phi)=\Phi^{-1}}{\longrightarrow} \tilde{L}=T_{m}(\Phi) L T_{m}(\Phi)^{-1}$,

(2) $L \stackrel{T_{n}(\bar{\Phi})=\partial_{q}^{-1} \bar{\Phi}}{\longrightarrow} \tilde{L}=T_{n}(\bar{\Phi}) L T_{n}(\bar{\Phi})^{-1}$,

then $\tilde{L}$ in the two cases above satisfies the q-mKP hierarchy: $\frac{\partial \tilde{L}}{\partial t_{k}}=\left[(\tilde{L})_{\geq 1}^{k}, \tilde{L}\right]$. 
Proof. The proof of the first case can be found in [4], thus here we only consider the second case.

$$
\begin{aligned}
\frac{\partial \tilde{L}}{\partial t_{k}} & =\partial_{q}^{-1} \frac{\partial \bar{\Phi}}{\partial t_{k}} \cdot L \cdot \bar{\Phi}^{-1} \partial_{q}+\partial_{q}^{-1} \bar{\Phi} \cdot \frac{\partial L}{\partial t_{k}} \cdot \bar{\Phi}^{-1} \partial_{q}-\partial_{q}^{-1} \bar{\Phi} \cdot L \cdot \bar{\Phi}^{-2} \frac{\partial \bar{\Phi}}{\partial t_{k}} \partial_{q} \\
& =\left[\partial_{q}^{-1} \bar{\Phi} \cdot L_{\geq 0}^{k} \cdot \bar{\Phi}^{-1} \partial_{q}-\partial_{q}^{-1} \cdot\left(L_{\geq 0}^{k}\right)^{*}(\bar{\Phi}) \cdot \bar{\Phi}^{-1} \partial_{q}, \partial_{q}^{-1} \bar{\Phi} \cdot L \cdot \bar{\Phi}^{-1} \partial_{q}\right]=\left[(\tilde{L})_{\geq 1}^{k}, \tilde{L}\right],
\end{aligned}
$$

where the second identity in Lemma 3.1 is used.

Corollary 3.1. Let $W, \Phi \neq \Phi_{1}, \bar{\Phi} \neq \bar{\Phi}_{1}$ be the dressing operator, eigenfunctions, adjoint eigenfunctions of the $q-K P$ hierarchy respectively, and $Z, \Psi, \bar{\Psi}$ be the ones for the $q-m K P$ hierarchy respectively, then under the anti-Miura transformations $T_{m}(\Phi)$ and $T_{n}(\bar{\Phi})$, the transformations of $W, \Phi_{1}, \bar{\Phi}_{1}$ are presented in the table below.

Table 1. Anti-Miura transformation $q-\mathrm{KP} \rightarrow q$ - $\mathrm{mKP}$

\begin{tabular}{cllll}
\hline$W \rightarrow Z$ & $Z=$ & $T=$ & $\Psi=$ & $\bar{\Psi}=$ \\
\hline$m$ & $\Phi^{-1} W$ & $\Phi^{-1}$ & $\Phi^{-1} \Phi_{1}$ & $\Phi \bar{\Phi}_{1}$ \\
$n$ & $\partial_{q}^{-1} \bar{\Phi} W \partial_{q}$ & $\partial_{q}^{-1} \bar{\Phi}$ & $\partial_{q}^{-1}\left(\bar{\Phi} \Phi_{1}\right)$ & $\partial_{q} \theta^{-1}\left(\bar{\Phi}^{-1} \bar{\Phi}_{1}\right)$ \\
\hline
\end{tabular}

Note that the anti-Miura transformation $T_{m}(\Phi)$ is also discussed in [4].

Proposition 3.2. There are two kinds of elementary Miura transformations $q-m K P \rightarrow q-K P$, that is, assume $Z$ to be the dressing operator of the $q-m K P$ hierarchy, and $z_{0}$ to be the coefficient of $\partial_{q}^{0}$-term in $Z$, then

(1) $\mu$ case: $W=z_{0}^{-1} Z$,

(2) $v$ case: $W=\theta\left(z_{0}^{-1}\right) \partial_{q} Z \partial_{q}^{-1}$,

satisfy $\frac{\partial W}{\partial t_{k}}=-\left(W \partial_{q}^{k} W^{-1}\right)_{<0} W$, i.e., $W$ is the dressing operator of the $q-K P$ hierarchy.

Proof. (1) $\mu$ case. Firstly, by using the first identity in Lemma 3.1, the comparison of $\partial_{q}^{0}$-term in (2.24) will lead to

$$
\frac{\partial z_{0}}{\partial t_{k}}=-z_{0}^{2}\left(W \partial_{q}^{k} W^{-1}\right)_{\geq 0}\left(z_{0}^{-1}\right)
$$

Further

$$
\frac{\partial z_{0}^{-1}}{\partial t_{k}}=\left(W \partial_{q}^{k} W^{-1}\right)_{\geq 0}\left(z_{0}^{-1}\right)
$$

Therefore $z_{0}^{-1}$ can be viewed as the eigenfunction of the $q$-KP hierarchy corresponding to the dressing operator $W=z_{0}^{-1} Z$. Then according to (2.24), (3.2) and the first identity in Lemma 3.1, one can find

$$
\begin{aligned}
\frac{\partial W}{\partial t_{k}} & =\frac{\partial}{\partial t_{k}}\left(z_{0}^{-1} Z\right)=\left(W \partial_{q}^{k} W^{-1}\right)_{\geq 0}\left(z_{0}^{-1}\right) \cdot Z-z_{0}^{-1}\left(z_{0} W \partial_{q}^{k} W^{-1} z_{0}^{-1}\right)_{\leq 0} Z \\
& =\left(W \partial_{q}^{k} W^{-1}\right)_{\geq 0}\left(z_{0}^{-1}\right) \cdot Z-\left(W \partial_{q}^{k} W^{-1}\right)_{<0} z_{0}^{-1} Z-\left(W \partial_{q}^{k} W^{-1}\right)_{+}\left(z_{0}^{-1}\right) \cdot Z \\
& =-\left(W \partial_{q}^{k} W^{-1}\right)_{<0} W .
\end{aligned}
$$


(2) $v$ case. By using the second identity in Lemma 3.1 and comparing the $\partial_{q}^{0}$-term in (2.24), one can derive

$$
\frac{\partial z_{0}}{\partial t_{k}}=-\theta^{-1}\left(\left(W \partial_{q}^{k} W^{-1}\right)_{\geq 0}^{*}\left(\theta\left(z_{0}\right)\right)\right)
$$

Then

$$
\frac{\partial \theta\left(z_{0}\right)}{\partial t_{k}}=-\left(W \partial_{q}^{k} W^{-1}\right)_{\geq 0}^{*}\left(\theta\left(z_{0}\right)\right)
$$

i.e., $\theta\left(z_{0}\right)$ is the adjoint eigenfunction of the $q$-KP hierarchy with respect to the dressing operator $W=\Lambda\left(z_{0}^{-1}\right) \partial_{q} Z \partial_{q}^{-1}$. By (2.24), (3.4) and the second identity in Lemma 3.1,

$$
\begin{aligned}
\frac{\partial W}{\partial t_{k}}= & \frac{\partial}{\partial t_{k}}\left(\theta\left(z_{0}^{-1}\right) \partial_{q} Z \partial_{q}^{-1}\right)=\theta\left(z_{0}^{-2}\right) \cdot\left(W \partial_{q}^{k} W^{-1}\right)_{\geq 0}^{*}\left(\theta\left(z_{0}\right)\right) \cdot \partial_{q} Z \partial_{q}^{-1} \\
& -\theta\left(z_{0}^{-1}\right) \partial_{q} \cdot\left(\partial_{q}^{-1} \theta\left(z_{0}\right) W \partial_{q}^{k} W^{-1} \theta\left(z_{0}^{-1}\right) \partial_{q}\right)_{\leq 0} Z \cdot \partial_{q}^{-1} \\
= & \theta\left(z_{0}^{-2}\right) \cdot\left(W \partial_{q}^{k} W^{-1}\right)_{\geq 0}^{*}\left(\theta\left(z_{0}\right)\right) \cdot \partial_{q} Z \partial_{q}^{-1}-\left(W \partial_{q}^{k} W^{-1}\right)_{<0} \cdot \theta\left(z_{0}^{-1}\right) \partial_{q} Z \partial_{q}^{-1} \\
& -\theta\left(z_{0}^{-1}\right) \cdot\left(W \partial_{q}^{k} W^{-1}\right)_{\geq 0}^{*}\left(\theta\left(z_{0}\right)\right) \cdot \theta\left(z_{0}^{-1}\right) \partial_{q} Z \partial_{q}^{-1} \\
= & -\left(W \partial^{k} W^{-1}\right)_{<0} W .
\end{aligned}
$$

Corollary 3.2. Assume $Z, \Psi, \bar{\Psi}$ to be the dressing operator, the eigenfunction and adjoint eigenfunction for the $q-m K P$ hierarchy respectively, and $W, \Phi, \bar{\Phi}$ to be the ones of the $q-K P$ hierarchy respectively, then under the Miura transformation, the changes of $Z, \Phi, \bar{\Phi}$ is showed in the following table.

Table 2. Miura transformation $q-\mathrm{mKP} \rightarrow q-\mathrm{KP}$

\begin{tabular}{ccccc}
\hline$Z \rightarrow W$ & $W=$ & $T=$ & $\Phi=$ & $\bar{\Phi}=$ \\
\hline$\mu$ & $z_{0}^{-1} Z$ & $z_{0}^{-1}$ & $z_{0}^{-1} \Psi$ & $z_{0} \bar{\Psi}$ \\
$v$ & $\theta\left(z_{0}^{-1}\right) \partial_{q} Z \partial_{q}^{-1}$ & $\theta\left(z_{0}^{-1}\right) \partial_{q}$ & $\theta\left(z_{0}^{-1}\right) \partial_{q}(\Psi)$ & $\theta\left(z_{0} \partial_{q}^{-1}(\bar{\Psi})\right)$ \\
\hline
\end{tabular}

Through $W \stackrel{\text { anti-Miura }}{\longrightarrow} Z \stackrel{\text { Miura }}{\longrightarrow} W^{\prime}$ and $Z \stackrel{\text { Miura }}{\longrightarrow} W \stackrel{\text { anti-Miura }}{\longrightarrow} Z^{\prime}$, one can obtain the autoBacklund transformations of the $q$-KP and $q$-mKP hierarchies. The corresponding results are summarized in Tables 3 and 4 . Here we let $\Phi_{1} \neq \Phi, \bar{\Phi}_{1} \neq \bar{\Phi}$ be the eigenfunctions and the adjoint eigenfunctions of the $q$-KP hierarchy respectively, and $\Psi_{1} \neq \Psi, \bar{\Psi}_{1} \neq \bar{\Psi}$ be the ones of the $q$-mKP hierarchy respectively.

Table 3. Auto-Backlund transformations $q-\mathrm{KP} \rightarrow q-\mathrm{KP}$

\begin{tabular}{ccccc}
\hline$W \rightarrow W^{\prime}$ & $W^{\prime}=$ & $T=$ & $\Phi_{1}^{\prime}=$ & $\bar{\Phi}_{1}^{\prime}=$ \\
\hline$m$ followed by $\mu$ & $W$ & 1 & $\Phi_{1}$ & $\bar{\Phi}_{1}$ \\
$n$ followed by $\mu$ & $\theta^{-1}\left(\bar{\Phi}^{-1}\right) \partial_{q}^{-1} \bar{\Phi} W \partial_{q}$ & $\theta^{-1}\left(\bar{\Phi}^{-1}\right) \partial_{q}^{-1} \bar{\Phi}$ & $\partial_{q}^{-1}\left(\bar{\Phi} \Phi_{1}\right) / \theta^{-1}(\bar{\Phi})$ & $\theta^{-1}(\bar{\Phi}) \cdot \partial_{q} \theta^{-1}\left(\bar{\Phi}_{1} / \bar{\Phi}\right)$ \\
$m$ followed by $v$ & $\theta(\Phi) \partial_{q} \Phi^{-1} W \partial_{q}^{-1}$ & $\theta(\Phi) \partial_{q} \Phi^{-1}$ & $\theta(\Phi) \cdot \partial_{q}\left(\Phi_{1} / \Phi\right)$ & $\theta\left(\partial_{q}^{-1}\left(\Phi \Phi_{1}\right) / \Phi\right)$ \\
$n$ followed by $v$ & $W$ & 1 & $\Phi_{1}$ & $\bar{\Phi}_{1}$ \\
\hline
\end{tabular}


Table 4. Auto-Backlund transformations $q$-mKP $\rightarrow q$-mKP

\begin{tabular}{cclll}
\hline$Z \rightarrow Z^{\prime}$ & $Z^{\prime}=$ & $T=$ & \multicolumn{1}{c}{$\Psi_{1}^{\prime}=$} & $\bar{\Psi}_{1}^{\prime}=$ \\
\hline$\mu$ followed by $m$ & $\Psi^{-1} Z$ & $\Psi^{-1}$ & $\Psi^{-1} \Psi_{1}$ & $\Psi \bar{\Psi}_{1}$ \\
$\nu$ followed by $m$ & $\partial_{q}(\Psi)^{-1} \partial_{q} Z \partial_{q}^{-1}$ & $\partial_{q}(\Psi)^{-1} \partial_{q}$ & $\partial_{q}\left(\Psi_{1}\right) / \partial_{q}(\Psi)$ & $\partial_{q}(\Psi) \cdot \theta \partial_{q}^{-1}\left(\bar{\Psi}_{1}\right)$ \\
$\mu$ followed by $n$ & $\partial_{q}^{-1} \bar{\Psi} Z \partial_{q}$ & $\partial_{q}^{-1} \bar{\Psi}$ & $\partial_{q}^{-1}\left(\bar{\Psi} \Psi_{1}\right)$ & $\partial_{q} \theta^{-1}\left(\bar{\Psi}_{1} / \bar{\Psi}\right)$ \\
$\nu$ followed by $n$ & $\partial_{q}^{-1} \cdot \theta \partial_{q}^{-1}(\bar{\Psi}) \cdot \partial_{q} Z$ & $\partial_{q}^{-1} \cdot \theta \partial_{q}^{-1}(\bar{\Psi}) \cdot \partial_{q}$ & $\Psi_{1} \partial_{q}^{-1}(\bar{\Psi})$ & $\partial_{q}\left(\partial_{q}^{-1}\left(\bar{\Psi}_{1}\right)\right.$ \\
& & & $-\partial_{q}^{-1}\left(\Psi_{1} \bar{\Psi}\right)$ & $\left./ \partial_{q}^{-1}(\bar{\Psi})\right)$ \\
\hline
\end{tabular}

Remark 1. Denote $T_{n \mu}$ as the $T$ derived through $n$ followed by $\mu$, and the others are the same. One can find that $T_{n \mu}$ and $T_{m v}$ are consistent with $T_{I}$ and $T_{D}$ in [6] respectively. $T_{\mu m}$ and $T_{v m}$ are the same as $T_{1}$ and $T_{2}$ in [4] respectively. $T_{\mu n}$ is a new auto-Backlund transformation for the $q$-mKP hierarchy. $T_{\mu m}, T_{v m}$ and $T_{\mu n}$ are the elementary auto-Backlund transformations for the $q$-mKP hierarchy. In fact, $T_{v n}$ can be obtained by $Z \stackrel{T_{v m}}{\longrightarrow} Z^{\prime} \stackrel{T_{\mu n}}{\longrightarrow} Z^{\prime \prime}$.

Remark 2. Because of the non-commutativity [4] for $T_{\mu m}, T_{v m}$ and $T_{\mu n}$ ( the non-commutativity of $T_{\mu n}$ can be proved easily by direct computation), they are not applicable in the $q$-mKP hierarchy. Thus it is necessary to seek other kinds of auto-Backlund transformations operators which can commute with each other in the $q$-mKP hierarchy. In fact, since 1 is one eigenfunction for the $q$-mKP hierarchy, one can define

$$
T_{D}(\Psi)=\partial_{q}\left(\Psi^{-1}\right)^{-1} \cdot \partial_{q} \cdot \Psi^{-1}, T_{I}(\bar{\Psi})=\partial_{q}^{-1}(\bar{\Psi})^{-1} \cdot \partial_{q}^{-1} \cdot \bar{\Psi}
$$

through $Z \stackrel{T_{\mu m}(\Psi)}{\longrightarrow} Z^{\prime} \stackrel{T_{v m}\left(1^{\prime}\right)}{\longrightarrow} Z^{\prime \prime}$ and $Z \stackrel{T_{\mu n}(\bar{\Psi})}{\longrightarrow} Z^{\prime} \stackrel{T_{\mu m}\left(1^{\prime}\right)}{\longrightarrow} Z^{\prime \prime}$ respectively. Here $T_{D}(\Psi)$ is the same as $T_{3}$ in [4], while $T_{I}(\bar{\Psi})$ is introduced for the first time. It can be proved that $T_{D}$ and $T_{I}$ commute with each other.

\section{Miura and auto-Backlund transformations for the $q$-cKP and $q$-cmKP hierarchies}

In Section 3, we have investigated the Miura and anti-Miura transformations for the $q$-KP and $q$ $\mathrm{mKP}$ hierarchies. Now we will extend these results to the the constrained cases. The crucial point is that the corresponding transformations must keep the forms of the Lax operators for the $q$-cKP and $q$-cmKP hierarchies (see (2.25) and (2.27)), which can be done through choosing the eigenfunctions in the corresponding constrained Lax operators. In fact, similar thoughts are firstly considered in the Darboux transformations of the constrained KP hierarchy [2].

In fact, if one chooses the eigenfunction $\Phi$ in the Lax operator $L_{q c K P}$ (see (2.25)), then according to the relation $\left(A_{\geq 0} f\right)_{[0]}=A_{\geq 0}(f)$, one can find that by (2.26)

$$
\begin{aligned}
& T_{m}(\Phi) L_{q c K P} T_{m}(\Phi)^{-1} \\
= & \Phi^{-1}\left(L_{q c K P}\right)_{\geq 0} \Phi+\Phi^{-1} \cdot\left(\Phi \partial_{q}^{-1} \bar{\Phi}\right) \cdot \Phi \\
= & \Phi^{-1} \theta^{N}(\Phi) \cdot \partial_{q}^{N}+\cdots+(\ln \Phi)_{t_{N}}+\partial_{q}^{-1} \bar{\Phi} \Phi
\end{aligned}
$$

has the form of the Lax operator for the $q$-cmKP hierarchy (see (2.27)) with

$$
v_{N}=\Phi^{-1} \theta^{N}(\Phi), v_{0}=(\ln \Phi)_{t_{N}}, \bar{\Psi}=\Phi \bar{\Phi} .
$$


Similarly by choosing the adjoint eigenfunction $\bar{\Phi}$ in $L_{q c K P}$ (see (2.25)) and using the relations $\left(\partial_{q}^{-1} A_{\geq 0} \partial_{q}\right)_{[0]}=\theta^{-1}\left(\left(A_{\geq 0}^{*}\right)_{[0]}\right)$ and $\left(\partial_{q}^{-1} A_{\geq 0}\right)_{<0}=\partial_{q}^{-1} \cdot\left(A_{\geq 0}^{*}\right)_{[0]}$, it can be proved that

$$
\begin{aligned}
& T_{n}(\bar{\Phi}) L_{q c K P} T_{n}(\bar{\Phi})^{-1} \\
= & \left(\partial_{q}^{-1} \bar{\Phi}\left(L_{q c K P}\right)_{\geq 0} \bar{\Phi}^{-1} \partial_{q}\right)_{\geq 0}+\left(\partial_{q}^{-1} \bar{\Phi}\left(L_{q c K P}\right)_{\geq 0} \bar{\Phi}^{-1} \partial_{q}\right)_{<0}+\partial_{q}^{-1} \bar{\Phi} \cdot \Phi \partial_{q}^{-1} \bar{\Phi} \cdot \bar{\Phi}^{-1} \partial_{q} \\
= & \theta^{-1}\left(\bar{\Phi} \theta^{N}\left(\bar{\Phi}^{-1}\right)\right) \cdot \partial_{q}^{N}+\cdots-\theta^{-1}(\ln \bar{\Phi})_{t_{N}}+\partial_{q}^{-1}\left(\partial_{q} \theta^{-1}\left((\ln \bar{\Phi})_{t_{N}}\right)+\bar{\Phi} \Phi\right)
\end{aligned}
$$

still has the form of (2.27) with

$$
v_{N}=\theta^{-1}\left(\bar{\Phi} \theta^{N}\left(\bar{\Phi}^{-1}\right)\right), v_{0}=-\theta^{-1}(\ln \bar{\Phi})_{t_{N}}, \bar{\Psi}=\partial_{q} \theta^{-1}\left((\ln \bar{\Phi})_{t_{N}}\right)+\bar{\Phi} \Phi
$$

These results are collected into Table 5 .

Table 5. Anti-Miura transformation $q$-cKP $\rightarrow q$-cmKP

\begin{tabular}{cccc}
\hline$L_{c q K P} \rightarrow L_{c q m K P}$ & $T=$ & $v_{0}=$ & $\bar{\Psi}=$ \\
\hline$m$ & $\Phi^{-1}$ & $(\ln \Phi)_{t_{N}}$ & $\Phi \bar{\Phi}$ \\
$n$ & $\partial_{q}^{-1} \bar{\Phi}$ & $-\theta^{-1}(\ln \bar{\Phi})_{t_{N}}$ & $\partial_{q} \theta^{-1}\left((\ln \bar{\Phi})_{t_{N}}\right)+\bar{\Phi} \Phi$ \\
\hline
\end{tabular}

On the other hand, for the Miura transformation, according to (2.27), one has

$$
T_{\mu} L_{q c m K P} T_{\mu}^{-1}=z_{0}^{-1}\left(L_{q c m K P}\right)_{+} z_{0}+z_{0}^{-1} \partial_{q}^{-1} \bar{\Psi}_{z_{0}}
$$

and

$$
\begin{aligned}
& T_{v} L_{q c m K P} T_{v}^{-1} \\
= & \left(\theta\left(z_{0}^{-1}\right) \partial_{q} L_{q c m K P} \partial_{q}^{-1} \theta\left(z_{0}\right)\right)_{\geq 0}+\left(\theta\left(z_{0}^{-1}\right) \partial_{q}\left(L_{q c m K P}\right)_{\leq 0} \partial_{q}^{-1} \theta\left(z_{0}\right)\right)_{<0} \\
= & \left(\theta\left(z_{0}^{-1}\right) \partial_{q} L_{q c m K P} \partial_{q}^{-1} \theta\left(z_{0}\right)\right)_{\geq 0}+\left(\theta\left(z_{0}^{-1}\right) \partial_{q}\left(v_{0}+\partial_{q}^{-1} \Psi\right) \partial_{q}^{-1} \theta\left(z_{0}\right)\right)_{<0} \\
= & \left(\theta\left(z_{0}^{-1}\right) \partial_{q} L_{q c m K P} \partial_{q}^{-1} \theta\left(z_{0}\right)\right)_{\geq 0}+\theta\left(z_{0}^{-1}\right)\left(\partial_{q}\left(v_{0}\right)+\Psi\right) \partial_{q}^{-1} \theta\left(z_{0}\right),
\end{aligned}
$$

which preserve the form of the Lax operator of the $q$-cKP hierarchy (see (2.25)). We summarize these results in Table 6.

Table 6. Miura transformation $q$-cmKP $\rightarrow q$-cKP

\begin{tabular}{clcl}
\hline$L_{c q m K P} \rightarrow L_{c q K P}$ & $T=$ & $\Phi=$ & $\bar{\Phi}=$ \\
\hline$\mu$ & $z_{0}^{-1}$ & $z_{0}^{-1}$ & $z_{0} \bar{\Psi}$ \\
$v$ & $\theta\left(z_{0}^{-1}\right) \partial_{q}$ & $\theta\left(z_{0}^{-1}\right)\left(\partial_{q}\left(v_{0}\right)+\bar{\Psi}\right)$ & $\theta\left(z_{0}\right)$ \\
\hline
\end{tabular}

Then $L_{q c K P} \stackrel{\text { anti-Miura }}{\longrightarrow} L_{q c m K P} \stackrel{\text { Miura }}{\longrightarrow} L_{q c K P}^{\prime}$ and $L_{q c m K P} \stackrel{\text { Miura }}{\longrightarrow} L_{q c K P} \stackrel{\text { anti-Miura }}{\longrightarrow} L_{q c m K P}^{\prime}$ give rise to the auto-Backlund transformations for the $q$-cKP and $q$-cmKP hierarchies. These results are listed in Table 7 and 8.

Table 7. Auto-Backlund transformations $q$-cKP $\rightarrow q$-cKP

\begin{tabular}{cccc}
\hline$L_{q c K P} \rightarrow L_{q c K P}^{\prime}$ & $T=$ & $\Phi^{\prime}=$ & $\bar{\Phi}^{\prime}=$ \\
\hline$m$ followed by $\mu$ & 1 & $\Phi$ & $\bar{\Phi}$ \\
$n$ followed by $\mu$ & $\theta^{-1}\left(\bar{\Phi}^{-1}\right) \partial_{q}^{-1} \bar{\Phi}$ & $\theta^{-1}\left(\bar{\Phi}^{-1}\right)$ & $\theta^{-1}(\bar{\Phi})\left(\bar{\Phi} \Phi+\partial_{q} \theta^{-1}(\ln \bar{\Phi})_{t_{N}}\right)$ \\
$m$ followed by $v$ & $\theta(\Phi) \partial_{q} \Phi^{-1}$ & $\theta(\Phi) \cdot\left(\Phi \bar{\Phi}+\partial_{q}(\ln \Phi)_{t_{N}}\right)$ & $\theta\left(\Phi^{-1}\right)$ \\
$n$ followed by $v$ & 1 & $\Phi$ & $\bar{\Phi}$ \\
\hline
\end{tabular}


Table 8. Auto-Backlund transformations $q$-cmKP $\rightarrow q$-cmKP

\begin{tabular}{lccc}
\hline$L_{q c m K P} \rightarrow L_{q c m K P}^{\prime}$ & $T=$ & $v_{0}^{\prime}=$ & $\bar{\Psi}^{\prime}=$ \\
\hline$\mu$ followed by $m$ & 1 & $v_{0}$ & $\bar{\Psi}$ \\
$v$ followed by $m$ & $\left(\bar{\Psi}+\partial_{q}\left(v_{0}\right)\right)^{-1} \partial_{q}$ & $\theta\left(v_{0}\right)+\ln \left(\partial_{q}\left(v_{0}\right)+\bar{\Psi}\right)_{t_{N}}$ & $\partial_{q}\left(v_{0}\right)+\bar{\Psi}$ \\
$\mu$ followed by $n$ & $\partial_{q}^{-1} \bar{\Psi}$ & $\theta^{-1}\left(v_{0}\right)-\theta^{-1}(\ln \bar{\Psi})_{t_{N}}$ & $\bar{\Psi}+\partial_{q} \theta^{-1}(\ln \bar{\Psi})_{t_{N}}-\partial_{q} \theta^{-1}\left(v_{0}\right)$ \\
$v$ followed by $n$ & 1 & $v_{0}$ & $\bar{\Psi}$ \\
\hline
\end{tabular}

In Table 8, the following fact is used.

$$
\frac{\partial \ln \left(z_{0}\right)}{\partial t_{N}}=-v_{0}
$$

which can be derived by the comparison of the $\partial_{q}^{0}$-term of the both sides in (2.24).

Remark 3. Different from the case of the $q$-mKP hierarchy in Table 4 , there are only two nontrivial auto-Backlund transformations for the $q$-cmKP hierarchy in Table 8, since the choices of $\Phi$ and $\bar{\Phi}$ for the anti-Miura transformation in Table 5 is unique due to keeping the form of (2.27).

Remark 4. The factor $\left(\bar{\Psi}+\partial_{q}\left(v_{0}\right)\right)^{-1}$ in Table 8 can be expressed as $\partial_{q}(\Psi)^{-1}$, where $\Psi \equiv \partial_{q}^{-1}(\bar{\Psi})+$ $v_{0}$ is the eigenfunction of the $q$-cmKP hierarchy, that is,

$$
\frac{\partial \Psi}{\partial t_{k}}=B_{k}(\Psi)
$$

with $\left(L_{q c m K P}^{k / N}\right)_{\geq 1}=B_{k}$. In fact from (2.28), one has

$$
\begin{aligned}
\frac{\partial v_{0}}{\partial t_{k}} & =\left[B_{k}, L_{q c m K P}\right]_{[0]}=\left[B_{k}, \partial_{q}^{-1} \bar{\Psi}+v_{0}\right]_{[0]} \\
& =\left(B_{k} \partial_{q}^{-1} \bar{\Psi}\right)_{[0]}+\left(B_{k} v_{0}\right)_{[0]}-\left(\partial_{q}^{-1} \bar{\Psi} B_{k}\right)_{[0]} \\
& =B_{k} \partial_{q}^{-1}(\bar{\Psi})+B_{k}\left(v_{0}\right)+\left(\partial_{q}^{-1} \cdot B_{k}^{*}\right)(\bar{\Psi}) \\
& =B_{k}\left(\partial_{q}^{-1}(\bar{\Psi})+v_{0}\right)-\partial_{q}^{-1}\left(\frac{\partial \bar{\Psi}}{\partial t_{k}}\right)
\end{aligned}
$$

which is just (4.4). Here $\left(\partial_{q}^{-1} A_{\geq 0} \partial_{q}\right)_{[0]}=\theta^{-1}\left(\left(A_{\geq 0}^{*}\right)_{[0]}\right)$ is used.

\section{Examples of Miura and auto-Backlund transformations}

In this section, we will give some examples of Miura and auto-Backlund transformations for the explicit equations in the $q$-KP and $q$-mKP hierarchies.

Firstly, let us derive the explicit Miura transformations from the $q$-mKP equation (2.20) to the $q$ KP equation (2.10). According to (2.12) and (2.22), one can express coefficients of $L_{q K P}$ and $L_{q m K P}$ in terms of the dressing operators $W$ and $Z$,

$$
\begin{gathered}
u=-(\theta-1)\left(w_{1}\right), \quad u_{1}=-\partial_{q}\left(w_{1}\right)-(\theta-1)\left(w_{2}\right)+w_{1}(\theta-1)\left(w_{1}\right), \cdots \\
v=z_{0} \theta\left(z_{0}^{-1}\right), \quad v_{0}=z_{0} \partial_{q}\left(z_{0}^{-1}\right)+z_{1} z_{0}^{-1}-\theta\left(z_{0}^{-1} z_{1}\right), \cdots .
\end{gathered}
$$


Then under the Miura transformation of $\mu$ type (see Table 2), one can get $w_{1}=z_{1} / z_{0}$. Further by (5.1) and (5.2)

$$
u=-(\theta-1)\left(w_{1}\right)=-\theta\left(z_{1} / z_{0}\right)+z_{1} / z_{0}=v_{0}-(q-1) x \cdot(v-1) .
$$

So by (2.17), one can get the Miura transformation of $\mu$ type

$$
u=(\theta-1)^{-1}\left(\frac{1}{v} \frac{\partial v}{\partial t_{1}}\right)-(q-1) x \cdot(v-1) .
$$

As for the Miura transformation of $v$ type (see Table 2), one has $w_{1}=\theta\left(z_{0}^{-1}\right) \partial_{q}\left(z_{0}\right)+\theta\left(z_{1} / z_{0}\right)$. Through (5.1), (5.2) and (2.17),

$$
\begin{aligned}
u & =-(\theta-1)\left(\theta\left(z_{0}^{-1}\right) \partial_{q}\left(z_{0}\right)\right)-\theta(\theta-1)\left(z_{1} / z_{0}\right) \\
& =\theta\left(\theta\left(z_{0}^{-1}\right) \partial_{q}\left(z_{0}\right)+z_{0} \partial_{q}\left(z_{0}^{-1}\right)\right)+\theta\left(v_{0}\right)+\theta\left(z_{0}^{-1}\right) \partial_{q}\left(z_{0}\right) \\
& =\theta\left(\partial_{q}\left(z_{0}^{-1} z_{0}\right)\right)+\theta\left(v_{0}\right)+(q-1) x \cdot \theta\left(z_{0}^{-1}\right) \cdot(\theta-1)\left(z_{0}\right) \\
& =\theta\left(v_{0}\right)+(q-1) x \cdot\left(1-\theta\left(z_{0}^{-1}\right) z_{0}\right) \\
& =\theta(\theta-1)^{-1}\left(\frac{1}{v} \frac{\partial v}{\partial t_{1}}\right)+(q-1) x \cdot(1-v) .
\end{aligned}
$$

(5.4) and (5.5) are the Miura transformations from the $q$-mKP equation (2.20) to the $q$-KP equation (2.10).

Next, we give the examples of the auto-Backlund transformations of the $q$-mKP equation (2.20) and the $q$-KP equation (2.10). For this, let us consider the following sequence of Miura and antiMiura transformations, starting from the vacuum solution $W=1$ and according to Table 1 and 2,

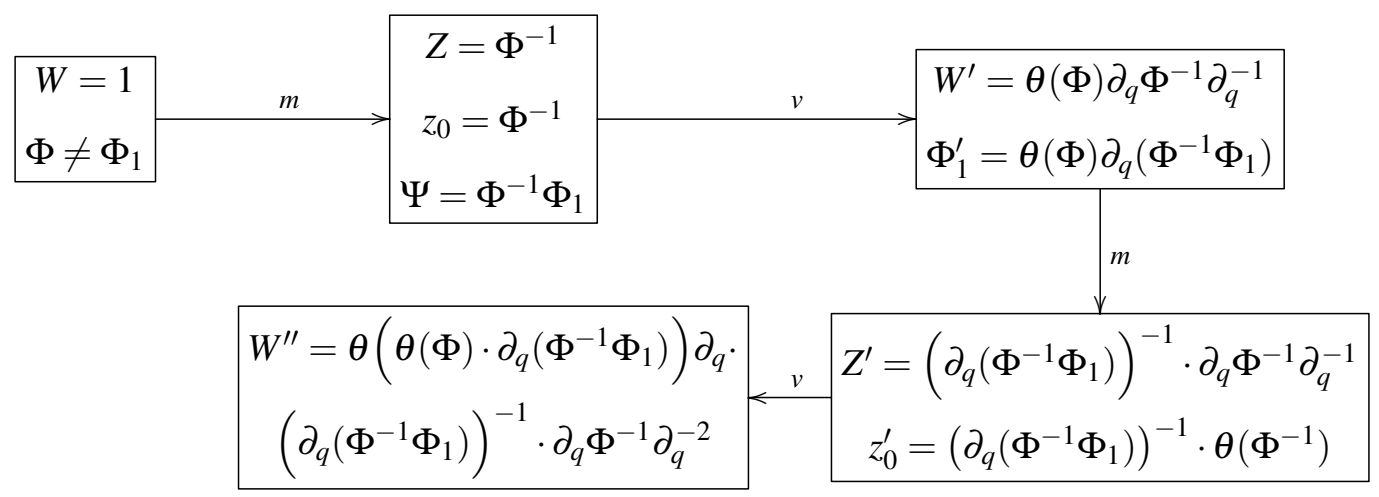

where $\Phi$ and $\Phi_{1}$ are two different eigenfunctions of the $q$-KP hierarchy corresponding to $W=1$. Based upon the sequence above, one can from (5.1) and (5.2) obtain the examples of the autoBacklund transformations of the $q$-mKP equation (2.20) and the $q$-KP equation (2.10),

$$
v=\theta(\Phi) / \Phi \stackrel{v \text { followed by } \mathrm{m}}{\longrightarrow} v^{\prime}=\theta\left(\theta(\Phi) \cdot \partial_{q}\left(\Phi^{-1} \Phi_{1}\right)\right) /\left(\theta(\Phi) \cdot \partial_{q}\left(\Phi^{-1} \Phi_{1}\right)\right) .
$$

and

$$
\begin{aligned}
& u=0 \stackrel{\mathrm{m} \text { followed by } v}{\longrightarrow} u^{\prime}=(\theta-1)\left(\partial_{q}(\Phi) / \Phi\right) \stackrel{\mathrm{m} \text { followed by } v}{\longrightarrow} \\
& u^{\prime \prime}=(\theta-1)\left((q-1) x \cdot\left(v^{\prime}-1\right)\right)+\theta(\theta-1)\left(\partial_{q}(\Phi) / \Phi\right) .
\end{aligned}
$$




\section{Conclusions and Discussions}

The Miura links between the $q$-KP and $q$-mKP hierarchies are established in Section 3. The antiMiura transformations from the $q$-KP hierarchy to the $q$-mKP hierarchies are showed in Table 1 . And the Miura transformations from the $q$-mKP hierarchy to the $q$-KP hierarchy are listed in Table 2 . Then the auto-Backlund transformations for the $q$-KP and $q$-mKP hierarchies are derived through the combinations of the Miura and anti-Miura transformations, which are presented in Table 3 and Table 4. The Miura and auto-Backlund transformations are also generalized to the constrained cases of the $q$-KP and $q$-mKP hierarchies in Section 4. The anti-Miura and Miura transformations for the $q$-cKP and $q$-cmKP hierarchies are given in Table 5 and Table 6 separately. And the corresponding auto-Backlund transformations are listed in Table 7 and Table 8. At last, some examples of the Miura and auto-Backlund transformations are given in Section 6. Here except the anti-Miura transformation $T_{m}$ also discussed in [4], the Miura and anti-Miura transformations in the $q$-deformed cases are completely new. And it is also new to obtain the auto-Backlund transformations in the $q$-deformed cases through the combinations of the Miura and anti-Miura transformations.

It is hoped that these results will be helpful in the further study of the inner structures and properties in the $q$-deformed integrable systems. Based upon the results in this paper, the successive applications of the auto-Backlund transformations for the $q$-mKP hierarchies could be considered in more comprehensive cases, besides the eigenfunction cases of $T_{\mu m}(\Psi), T_{v m}(\Psi)$ and $T_{D}(\Psi)$ given in [4].

\section{Acknowledgments}

This work is supported by the Fundamental Research Funds for the Central Universities (Grant No. 2015QNA43). Many thanks to the anonymous referee for his/her useful comments.

\section{References}

[1] M. Adler, E. Horozov and P. van Moerbeke, The solution to the $q$-KdV equation, Phys. Lett. A 242(1998) 139-151.

[2] H. Aratyn, E. Nissimov and S. Pacheva, Virasoro symmetry of constrained KP hierarchies, Phys. Lett. A 228 (1997) 164-175.

[3] R. Carroll, Versions of quantum KdV, Int. J. Pure App. Math. 5 (2003) 173-206.

[4] J. P. Cheng, J. Z. Wang and X. Y. Zhang, The gauge transformation of the $q$-deformed modified KP hierarchy, J. Nonlin. Math. Phys. 21 (2014) 533-542.

[5] E. Frenkel, Deformations of the KdV hierarchy and related soliton equations, Int. Math. Res. Not. 2 (1996) 55-76.

[6] J. S. He, Y. H. Li and Y. Cheng, $q$-deformed KP hierarchy and $q$-Deformed constrained KP hierarchy, SIGMA 2 (2006) 060.

[7] P. Iliev, Tau function solutions to a $q$-deformation of the KP hierarchy, Lett. Math. Phys. 44 (1998) 187-200.

[8] V. Kac and P. Cheung, Quantum Calculus (Springer-Verlag, New York, 2002).

[9] B. G. Konopelchenko, On the gauge-invariant description of the evolution equations integrable by Gelfand-Dikij spectral problems, Phys. Lett. A. 92 (1982) 323-327.

[10] B. A. Kupershmidt, Canonical property of the Miura maps between the MKP and KP hierarchies, Continuous and Discrete, Commun. Math. Phys. 167 (1995) 351-371.

[11] C. Z. Li, Sato theory on the $q$-Toda hierarchy and its extension, Chaos, Solitons \& Fractals 76 (2015) 10-23.

[12] R. L. Lin, X. J. Liu and Y. B. Zeng, A new extended $q$-deformed KP hierarchy, J. Nonlin. Math. Phys. 15(2008) 333-347. 
[13] R. L. Lin, H. Peng and M. Manas, The $q$-deformed mKP hierarchy with self-consistent sources, Wronskian solutions and solitons, J. Phys. A: Math. Theor. 43 (2010) 434022.

[14] S. Majid, Foundations of quantum group theory (Cambridge University Press, Cambridge, 1995).

[15] R. M. Miura, Korteweg-de Vries equation and generalizations. I. a remarkable explicit nonlinear transformation, J. Math. Phys. 9 (1968) 1202-1204.

[16] W. Oevel and W. Strampp, Constrained KP hierarchy and bi-Hamiltonian structures, Commun. Math. Phys. 157 (1993) 51-81.

[17] J. C. Shaw and M. H. Tu, Miura and auto-Backlund transformations for the cKP and cmKP hierarchies, J. Math. Phys. 38 (1997) 5756-5773.

[18] K. Takasaki, $q$-Analogue of modified KP hierarchy and its quasi-classical Limit, Lett. Math. Phys. 72 (2005) 165-181.

[19] K. L. Tian, J. S. He, Y. C. Su and Y. Cheng, String equations of the $q$-KP hierarchy, Chin. Ann. Math. B 32 (2011) 895-904.

[20] K. L. Tian, J. S. He and Y. C. Su, Symmetric $q$-deformed KP hierarchy, Chin. Ann. Math. B 36 (2015) $1-10$.

[21] M. H. Tu, J. C. Shaw, and C. R. Lee, On Darboux - Backlund transformations for the $q$-deformed Korteweg - de Vries hierarchy, Lett. Math. Phys. 49 (1999) 33 - 45.

[22] M. H. Tu, $q$-deformed KP hierarchy: its additional symmetries and infinitesimal Backlund transformations, Lett. Math. Phys. 49 (1999) 95-103.

[23] M. H. Tu, J. C. Shaw and C. R. Lee, On the $q$-deformed modified Korteweg-de Vries hierarchy, Phys. Lett. A 266 (2000) 155-159.

[24] S. K. Wang, K. Wu. X. N. Wu and D. L. Yu, The q-deformation of AKNS-D hierarchy, J. Phys. A: Math. Gen. 34 (2001) 9641-9651. 